\title{
Celebrating women in science
}

\author{
Open discourse to identify challenges and devise solutions is essential to abolish gender inequalities globally and in \\ science. In our 'Focus on Women in Science', we celebrate the achievements and consider the concerns of women \\ researchers from around the world, who share some of the turning points of their scientific careers.
}

O ne of the world's greatest paradoxes is that, although women represent roughly $50 \%$ of the global

population, gender inequality persists to this day. Despite marked advances towards bridging disparities during the twentieth century, progress has been slow. According to data from the United Nations' (UN) Gender Inequality Index (a measure of gender differences in reproductive health, empowerment and economic status), women remain globally disadvantaged when it comes to reproductive health, representation in government, access to education and participation in the labour market ${ }^{1}$. Although more economically advanced countries score higher for health and education than the developing world, they too display large gender gaps in the proportion of women in government and the workforce. Recognizing this major societal challenge, world leaders have pledged to increase efforts to tackle it: achieving gender equality and empowering women and girls constitutes Goal 5 of the 17 Sustainable Development Goals of the 2030 Agenda for Sustainable Development adopted in a historic UN summit in September $2015^{2}$.

Unfortunately, science is not immune to these inequalities. UNESCO's Science Report Towards 2030 notes that women only make up $\sim 30 \%$ of the global researcher pool ${ }^{3}$. The same report describes a disconcerting 'leaky pipeline' when it comes to women in research. Although they represent $53 \%$ of bachelor's and master's degree holders, their numbers decline to $43 \%$ of $\mathrm{PhD}$ graduates, followed by a dramatic drop to $28 \%$ at the researcher level. More granular geographic analysis of the data shows that some regions, such as parts of eastern Europe, Latin America and Southeast Asia, have in fact achieved, or are close to achieving, gender parity. However, some of the world's most economically advanced countries display worryingly low numbers of female researchers. For example, women make up only $33 \%$ of researchers in the European Union (EU), with France and Germany displaying a low $25 \%$. Shockingly, only $15 \%$ of researchers in Japan are female, and a paltry $18 \%$ in South Korea. The low proportion of women in research closely parallels their limited share of parliamentary and managerial positions (both $\sim 30 \%$ according to UN data ${ }^{4}$ ).
Matters do not improve for those women who do pursue science careers. Implicit bias stemming from deeply rooted gender stereotypes may lead to disparities in the opportunities offered to female versus male students ${ }^{5}$. This imbalance translates to the higher echelons of research, with only $20 \%$ of full professors being women in the $\mathrm{EU}^{6}$. Additionally, of the 923 Nobel laureates since 1901, only 48 have been women. Nineteen received their awards between 2000-2017, representing only 9\% of Nobel prize recipients in the twentyfirst century. Women scientists also face a substantial gender pay gap, as highlighted by recent salary analyses of science-related employers in the $\mathrm{UK}^{7}$ and $\mathrm{PhD}$ holders in the US ${ }^{8}$. Worse is the increased likelihood of being subjected to sexual harassment. The National Academies of Sciences, Engineering and Medicine in the US recently reported that $20-50 \%$ of women students, faculty and staff in academic sciences, engineering and medicine, stated in various relevant surveys that they had been harassed ${ }^{9}$. It is necessary to further scrutinize what prevents so many women from pursuing research careers, and why those who do are not always given merit-based rewards and equal opportunities for career progression, and may be subjected to gender-based discriminatory behaviours.

A first essential step in our collective efforts to bridge the gender gap and foster equality is to listen to women researchers, understand their experiences, consider their concerns and celebrate their successes. To that end, we are proud to present our 'Focus on Women in Science, in which thirteen women scientists recount personal stories and key moments in their scientific careers. The Turning Point essays in this issue are also collected in a dedicated website of the journal (https://www. nature.com/collections/fyfvkbpslf/).

In these essays, our readers will find a convergence of themes. This was unplanned as the topic was non-prescriptive, but it is perhaps an unsurprising outcome. Indeed, some themes will resonate with all scientists irrespective of gender: moving far away from home in the pursuit of research; navigating the complex $\mathrm{PhD}$, postdoc and faculty landscapes; persevering in the face of setbacks in research projects and lab experiences; making difficult career choices in academia and industry; finding supportive peers and mentors. Other topics, such as the casual or more explicit sexism that still permeates many facets of society and research environments, are more specific to women. According to UN data, women do three times more unpaid care and domestic work than men $^{4}$. Many female readers will thus be familiar with another common theme: the decision of whether and when to start a family, and the need for fair, inclusive family policies and support. But if there is a truly unifying thread that connects these thirteen stories, it is the strength, passion and honesty of the authors, who offer valuable advice: do not give up; seek and offer mentorship and collaboration; have the confidence to seize opportunities and follow your heart. Some messages stand out: speak up in the face of bias and impropriety; give back to society by advocating for trainees, patients and science policy; work to bring about change.

In putting together this Focus issue, our aim was to provide a snapshot of the research world through the eyes of women scientists from diverse scientific backgrounds, offering a pluralism of opinions and experiences. We are grateful to our authors for sharing their stories, and we only regret that space and time constraints have not permitted the inclusion of a larger number of essays. Finally, we hope that our readers will find them inspirational and thought-provoking additions to the discussion on diversity and equality in science.

Published online: 28 August 2018 https://doi.org/10.1038/s41556-018-0190-4

References

1. Human Development Reports: Table 5 Gender Equality Index (United Nations Development Program, 2016).

2. United Nations Sustainable Development Goals https://go.nature. $\mathrm{com} / 2 \mathrm{M} 167 \mathrm{oB}$ (2015).

3. UNESCO Science Report Towards 2030 (United Nations Educational, Scientific and Cultural Organisation, 2016).

4. Spotlight on Sustainable Development Goal 5: Achieve gender equality and empower all women and girls. UN Women https:// go.nature.com/2KCg9XI (2017).

5. Proc. Natl Acad. Sci. USA 109, 16474-16479 (2012).

6. She Figures 2012: Gender in Research and Innovation (European Commission, 2012).

7. Nature 556, 160 (2018)

8. Survey of Earned Doctorates (National Science Foundation, 2016); https://go.nature.com/2OooGQv

9. National Academies of Sciences, Engineering and Medicine. Sexual Harrassment of Women: Climate, Culture and Consequences in Academic Sciences, Engineering and Medicine (National Acadamies Press, Washington DC, 2018). 